\title{
HEMOGLOBINA GLICOSILADA UN FACTOR ALIADO EN LA PREVENCIÓN DE INFECCIÓN POSTQUIRÚRGICA DEL PIE DIABÉTICO
}

\author{
GLYCOSILED HEMOGLOBIN FACTOR ALLIED IN THE PREVENTION OF POST-SURGICAL \\ INFECTION OF THE DIABETIC FOOT \\ Angela Marcela Villanueva-Del Busto ${ }^{1, a}$, Fiorella Rocío Arce-Huaroto ${ }^{1, a}$
}

\begin{abstract}
Sr. Editor
La diabetes mellitus tipo 2 es un problema de salud pública en el Perú y en el mundo. Según la Federación Internacional de Diabetes, se estima que existen en el mundo 387 millones de personas con DM-2. Según estudios a nivel nacional, la prevalencia de DM-2 en el Perú se encuentra entre el 4,1\% y 8,4\%. La principal complicación crónica de la DM-2, tanto por su frecuencia como por la consecuente discapacidad que genera, es el pie diabético. Así, $15 \%$ de los pacientes diabéticos desarrollarán lesiones en los pies. La mayoría de ellos sufrirán una epitelización exitosa de sus úlceras pero entre el $15 \%$ y $20 \%$, sufrirán amputaciones del miembro inferior ${ }^{(1)}$.
\end{abstract}

Al respecto, se sabe que los pacientes con diabetes mellitus tienen un alto riesgo de complicaciones postoperatorias, incluyendo infecciones, curación inadecuada de heridas, eventos cardiovasculares, tromboembolismo venoso, y la mortalidad. Debido a que se ha pensado que la hiperglucemia media este riesgo, se suele recomendar optimizar el control glicémico, en casos de cirugía electiva ${ }^{(2)}$.

El control glucémico deficiente, indicado por los niveles elevados de hemoglobina glicosilada (HbA1c), podría asociarse a un mayor riesgo de complicaciones postoperatorias. Existen diferentes estudios que han evaluado el efecto de los niveles de $\mathrm{HbA} 1 \mathrm{c}$ en el desarrollo de las infecciones del sitio quirúrgico, proponiendo una relación de causa efecto entre los mismos.

Nuestro objetivo fue revisar la evidencia con respecto al efecto de la HbA1c en el desarrollo de infección postquirúrgica en paciente con pie diabético.

Se realizó la búsqueda electrónica de diversos artículos científicos relacionados con el tema. Las fuentes de búsqueda fueron PubMed, Scielo, BVS, Scholar google y el Repositorio de la Universidad Ricardo Palma. Las palabras clave fueron: "glycosylated hemoglobin", "Diabetic foot infection", "infectious complication" $y$ "Amputee diabetic foot", "hemolobina glicosilada", "infección de pie diabético amputado", "marcadores de infección postquirúrgica del pie diabético".

Se encontraron 5 artículos que evalúan esta asociación (tabla 1), todos ellos de naturaleza retrospectiva, en 4 de ellos se encontró asociación estadística significativa, con OR que fluctuó entre 3,94 y 1,05, mientras que en 1 de ellos no se encontró asociación significativa entre ambos factores.

A pesar de que la mayoría de los estudios encuentra una asociación estadísticamente significativa entre los niveles elevados de hemoglobina glicosilada prequirúrgica y el desarrollo de infecciones postoperatorias, la calidad de evidencia puede ser cuestionable, por la naturaleza retrospectiva. Por otro lado, al no haber encontrado estudios nacionales no se puede hacer conclusiones sólidas para nuestra población, por o cual se propone la realización de los mismos que reflejen la problemática nacional. Ya que, de comprobarse la relación, los pacientes con niveles incrementados de hemoglobina glicosilada preoperatoria deberán ser evaluados apropiadamente y monitorizar continuamente la evolución de la herida mediante un equipo multidisciplinario, a fin de evitar complicaciones.

Universidad Ricardo Palma, Lima-Perú

astudiante de la Facultad de Medicina Humana.

Citar como: Angela Marcela Villanueva-Del Busto, Fiorella Rocío Arce-Huaroto. Hemoglobina glicosilada un factor aliado en la prevención de infección postquirúrgica del pie diabético. Rev. Fac. Med. Hum. Enero 2020; 20(1):162-163. DOI 10.25176/RFMH.v20i1.2198 
Tabla 1. Características generales de los artículos seleccionados.

\begin{tabular}{|c|c|c|c|}
\hline Autor año & $\begin{array}{l}\text { Tamaño de } \\
\text { muestra }\end{array}$ & Diseño de estudio & Resultados \\
\hline $\begin{array}{l}\text { Domek }^{(2)} \\
2016\end{array}$ & 21854 pacientes & $\begin{array}{l}\text { Estudio retrospectivo } \\
\text { observacional de serie de } \\
\text { casos }\end{array}$ & $\begin{array}{l}\text { El valor de la } \mathrm{HbA} 1 \mathrm{c} \text { en quienes desarrollaron } \\
\text { infección tuvo un promedio de } 6,41 \% \text {, en } \\
\text { comparación de un } 6,11 \% \text { para quienes no } \\
\text { desarrollaron la infección (OR } 1,05 \text { IC95\% } \\
\mathrm{P}=0,015 \text { ) }\end{array}$ \\
\hline $\begin{array}{l}\text { Cancienne(3) }^{(3)} \\
2017\end{array}$ & 7736 pacientes & $\begin{array}{l}\text { Análisis de base de una } \\
\text { serie de casos }\end{array}$ & $\begin{array}{l}\text { Los pacientes con un nivel de } \mathrm{HbA} 1 \mathrm{c} \text { de } 7,5 \% \\
\text { o más, tenían un riesgo significativamente } \\
\text { mayor de infección en comparación con } \\
\text { pacientes por debajo de este umbral (OR, } \\
2,6 \text {; IC } 95 \%, 1.9-3.4 ; \mathrm{P}<0,0001 \text { ) }\end{array}$ \\
\hline $\begin{array}{l}\text { Muñoz } z^{(4)} \\
2003\end{array}$ & 740 pacientes & $\begin{array}{l}\text { Estudio de cohortes } \\
\text { históricas }\end{array}$ & $\begin{array}{l}\text { No existe correlación entre el grado } \\
\text { de control glucémico }(\mathrm{HbA} 1 \mathrm{c} \%) \text { con la } \\
\text { frecuencia de infecciones en los pacientes } \\
\text { diabéticos Estudiados }(p=0,33)\end{array}$ \\
\hline $\begin{array}{l}\text { Jupiter(5) } \\
2014\end{array}$ & 322 pacientes & $\begin{array}{l}\text { Estudio retrospectivo } \\
\text { observacional de una } \\
\text { base de datos }\end{array}$ & $\begin{array}{l}\text { La tasa de infección aumentó de manera } \\
\text { constante a medida que la HbA1c aumentó } \\
\text { hasta } 7.3 \% \text {. y luego lentamente hasta que la } \\
\text { HbA1c alcanzó un } 8 \% \text { a un } 8,5 \% \text {, mientras } \\
\text { que aumentó drásticamente cuando la } \\
\text { HbA1c llego a niveles de un } 10 \% \text { (OR } 3,94 \text { IC } \\
95 \%: 2,38 \text { a } 6,61 \text { ) }\end{array}$ \\
\hline $\begin{array}{l}\text { Humphers(6) } \\
2014\end{array}$ & 322 pacientes & $\begin{array}{l}\text { Estudio de cohorte } \\
\text { retrospectivo }\end{array}$ & $\begin{array}{l}\text { La HbA1c se asoció significativamente con } \\
\text { las infecciones postoperatorias., con cada } \\
\text { incremento del } 1 \% \text { de HbA1c aumenta las } \\
\text { probabilidades de infección en un factor de } \\
1.59 \text { y de complicaciones en la cicatrización } \\
\text { de la herida }(\mathrm{OR}=1,25 \text {, IC } 95 \%=1,02-1,53)\end{array}$ \\
\hline
\end{tabular}

Contribuciones de autoría: Los autores participaron

Conflicto de interés: Los autores declaran no tener en la concepción, recolección de información, redacción y aprobación de la versión final del artículo.

Financiamiento: Autofinanciado. conflictos de intereses en la publicación de este artículo.

Recibido: 15 de noviembre del 2019

Aprobado: 26 de diciembre del 2019

Correspondencia: Angela Marcela Villanueva Del Busto.

Dirección: Jr. Vulcano Mz. D Lot 1. Urb. Santa Modesta, Santiago de Surco, lima-Perú.

Teléfono: 987758961

Correo: marcelavillanuevadelbusto@gmail.com

\section{REFERENCIAS BIBLIOGRÁFICAS}

1. Ministerio de Salud. Guía técnica: Guía de práctica clínica para el diagnóstico tratamiento y control del pie diabético. Resolución Minist - Perú [Internet] 2016;1-16. Available from: http://bvs.minsa.gob.pe/local/MINSA/3971.pdf

2. Domek N, Dux K, Pinzur M, Weaver F, Rogers T. Association Between Hemoglobin A1c and Surgical Morbidity in Elective Foot and Ankle Surgery. J Foot Ankle Surg [Internet]. 2016;55(5):939-43. Available from: http://dx.doi. org/10.1053/j.jfas.2016.04.009

3. Cancienne JM, Werner BC, Browne JA. Is There a Threshold Value of Hemoglobin A1c That Predicts Risk of Infection Following Primary Total Hip Arthroplasty? J Arthroplasty [Internet]. 2017;32(9):S236-40. Available from: http://dx.doi.org/10.1016/j.arth.2017.01.022

4. M. C. Martín Muñoz, A. Gómez de la Cámara, a. Román Martínez, p. Ferrando Vivas, M. E. Albarrán Juan Fh. Riesgo de infecciones y control metabólico en pacientes con diabetes mellitus tipo 2. An Med Interna [Internet]. 2004;21(3):28043. Available from: http://scielo.isciii.es/scielo.php?script=sci_ arttext\&pid=S0212-71992004000300004

5. Jupiter DC, Humphers JM, Shibuya N. Trends in postoperative infection rates and their relationship to glycosylated hemoglobin levels in diabetic patients undergoing foot and ankle surgery. J Foot Ankle Surg [Internet]. 2014;53(3):307-11. Available from: http://dx.doi.org/10.1053/j. jfas.2013.10.003

6. Humphers JM, Shibuya N, Fluhman BL, Jupiter D. The impact of glycosylated hemoglobin and diabetes mellitus on wound-healing complications and infection after foot and ankle surgery. J Am Podiatr Med Assoc [Internet]. 2014;104(4):320-9. Available from: http://www.ncbi.nlm.nih.gov/ pubmed/25076074 